\title{
An Internet DGPS Service for Precise Outdoor Navigation
}

\author{
Manuel G. Soares \\ Dept. of Electrical Engineering, \\ ISEP, \\ Rua Dr. António Bernardino de \\ Almeida, 431, \\ P-4200-072 Porto \\ PORTUGAL
}

\author{
Benedita Malheiro \\ Dept. of Electrical Engineering, \\ ISEP, \\ Rua Dr. António Bernardino de \\ Almeida, 431, \\ P-4200-072 Porto \\ PORTUGAL
}

\author{
Francisco J. Restivo \\ Dept. of Electrical Engineering \\ and Computers, FEUP, \\ Rua Dr. Roberto Frias, \\ P-4200-465 Porto \\ PORTUGAL
}

\begin{abstract}
The goal of the work presented in this paper is to provide mobile platforms within our campus with a GPS based data service capable of supporting precise outdoor navigation. This can be achieved by providing campus-wide access to real time Differential GPS (DGPS) data.

As a result, we designed and implemented a three-tier distributed system that provides Internet data links between remote DGPS sources and the campus and a campus-wide DGPS data dissemination service. The Internet data link service is a two-tier client/server where the server-side is connected to the DGPS station and the client-side is located at the campus. The campus-wide DGPS data provider disseminates the DGPS data received at the campus via the campus Intranet and via a wireless data link. The wireless broadcast is intended for portable receivers equipped with a DGPS wireless interface and the Intranet link is provided for receivers with a DGPS serial interface. The application is expected to provide adequate support for accurate outdoor campus navigation tasks.
\end{abstract}

\section{INTRODUCTION}

The need to improve the accuracy of the position readings obtained with standard Global Positioning System (GPS) receivers in outdoor navigation tasks carried at our campus led to the research and development described in this paper.

One solution to improve the accuracy of GPS measurements is the joint use of Global Positioning System data and Differential Global Positioning System data. Receivers are then able to correlate data from both sources (the GPS satellite data and the DGPS correction data), granting users higher accuracy readings.

However, while the availability of worldwide continuous GPS data (three-dimensional position, velocity and time) to users is guaranteed by the GPS service, the same is not true with appropriate DGPS data sources. The availability and adequacy of DGPS data sources depends, not only, on the existence of nearby DGPS base stations the validity and accuracy of the corrections depend on the distance between base station and rover, but also, on appropriate data dissemination over the area under consideration.

As a result, in order to increase the positioning accuracy obtained with GPS receivers within our campus, appropriate real time DGPS data must be provided. To solve this problem we designed and implemented a three-tier distributed system that establishes Internet data links between DGPS sources and the campus and provides a campus-wide DGPS data dissemination service.

This paper describes in detail this project. Section 1 introduces the reader to our motivation and goal. Section 2 provides a brief description of the features of the GPS and DGPS systems. Section 3 presents the developed application and section 4 describes the current status of the project. Section 5 reports on related work and section 6 presents the conclusions.

\section{REAL TIME POSITIONING}

Nowadays, real time positioning is an activity supported by satellite-based systems, namely, the North-American NAVSTAR Global Positioning System (GPS) and the Russian Global Navigation Satellite System (GLONASS). Both systems provide continuous, worldwide, three-dimensional position, velocity and time information to end-users. In the near future, the European GALILEO satellite radio navigation system is expected to become the first non-military real time positioning system.

GPS utilises the concept of time-of-arrival (TOA) ranging to determine the user position. This concept entails measuring the time a signal transmitted by an emitter at a known location takes to reach a user receiver. This time interval, referred to as the signal propagation time, is then multiplied by the speed of the signal (the speed of light) to obtain the emitter-to-receiver distance. By measuring the propagation time of signais broadcasted from multiple emitters at known locations (the GPS satellites), the receiver can determine its position.

\section{A Differential Global Positioning System}

The permanent quest for higher position readings accuracy led to the development of a GPS subsystem called the Differential Global Positioning System (DGPS). By definition, the DGPS method uses well-known geographic locations as references to detect the range errors of the GPS satellites. The method relies on a set of stations, called DGPS base stations, equipped with elaborated GPS receivers ( 12 channel single or dual receivers) and situated at precisely geo-referenced locations, to compute and 
broadcast the corrections to be applied to the GPS measurements (Fig. 1). With base stations and rovers recording observations at the same time, GPS processing software can reduce or eliminate "common errors" (such as the variable delays introduced in the GPS signal when it crosses the ionosphere and the troposphere) and by the ephemeris and clock errors of the GPS satellites

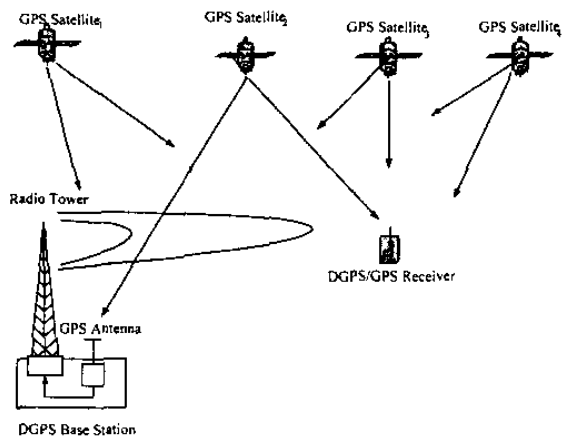

Fig. 1 - Components of a DGPS System.

There are two real time differential positioning methods: code phase and carrier phase. DGPS utilizing code phase measurements - pseudorange corrections - can provide a relative accuracy of a few meters $(1 \mathrm{~m}$ to $3 \mathrm{~m}$ ). DGPS utilizing carrier phase measurements - real time kinematic (RTK) corrections - can provide a relative accuracy of a few centimetres.

The receiving equipment and the access rules applied to the different types of DGPS data networks vary. While the DGPS data transmitted via radio beacon is public, free and complies with standard commercial DGPS/GPS receivers, DGPS data broadcasted via satellite is proprietary, requires the payment of an annual fee and the use of specific receiver equipment. The access to Internet links depends on the policy adopted by the service provider and is intended for standard commercial DGPS/GPS receivers.

\section{DESIGN AND IMPLEMENTATION}

The developed distributed system implements two main tasks: the establishment of the Internet data links between the DGPS sources and the ISEP campus and the campus-wide dissemination of the DGPS data.

The overall application is a three-tier client/server distributed application. The first tier - the client tier represents the end client applications (wireless or Intranet clients located within the campus). The intermediate layer - the campus server - fetches and disseminates the DGPS data for the end client applications. The third layer - the servers of DGPS data - acts as the data provider layer to the intermediate layer.

The first and third layers are insulated. This approach prevents the potential congestion of the data source servers and allows the adoption of different transport protocols between the first and second layers and between the second and first layers. In Fig. 2 we present an overview of the system's architecture.

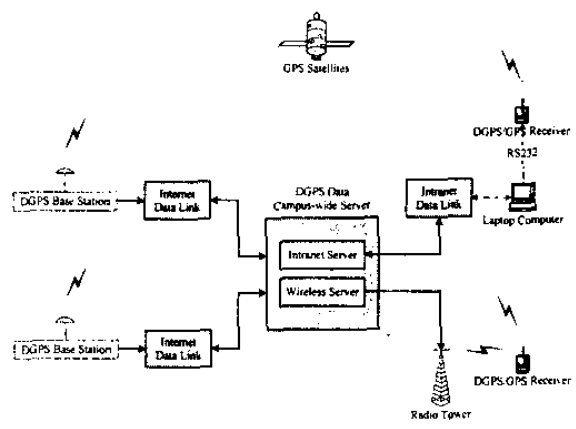

Fig. 2 - Overview of the System's Architecture.

The application provides three types of data links: Intranet and wireless data links - between the first and second layers - and Internet data links - between the second and third layers.

\section{A DGPS Internet Data Link Application}

The Internet link is accomplished through a two-tier client/server application. The server application, located at the third tier, is installed in a host at the DGPS base station and is continuously fed by the DGPS data generated by the DGPS base station. The interface between the DGPS base station equipment and the DGPS data link server application is controlled by the DGPS base station equipment and consists of a RS232 interface. The data link client application, which is located at the intermediate tier, has a double role: it acts as the Internet data link client and as the input module of the campus-wide data provider.

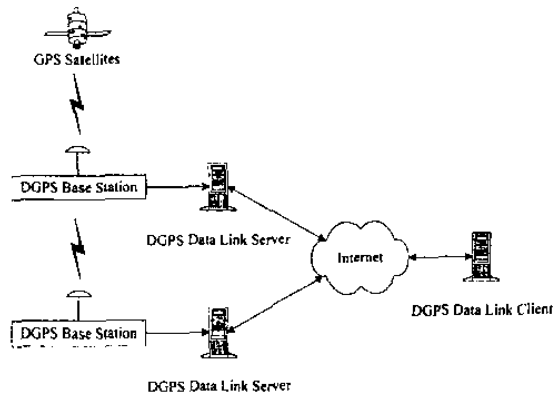

Fig. 3 - DGPS Internet Data Link Two-tier Application.

The implemented DGPS Internet Data Link application (Fig. 3) provides two types of data links: a full-duplex, point-to-point, connection-oriented and a simplex, point-to-multipoint, connectionless type of communication. While the first link relies on the transport layer's Transmission Control Protocol (TCP) to provide a reliable communication service, the second type of link uses the transport layer's User Datagram Protocol (UDP) to provide a multicast message-oriented service. Additionally, both links allow multithreading, i.e., it is possible to use the 
server-side application to provide, simultaneously, data to multiple clients.

In our case, since we intend to use multiple DGPS data sources, the client application can, simultaneously, connect to different DGPS data servers. Each server-side application data link is uniquely identified by its port and IP address. When the service link is supported by TCP, the IP address is the host IP address but when the service link supported by UDP, the IP address is the selected class D IP address (class D IP addresses are in the range 224.0.0.0 to 239.255.255.255, inclusive). The port number assigned by the Internet Assigned Numbers Authority (IANA) for the dissemination of DGPS correction data is port 2101.

\section{A.I Data Transmission}

The developed Internet Data Link Application allows data transmission over TCP (unicast) and UDP (multicast). The data transmission can be implemented using data frames (frame mode) or, simply, bytes (raw mode). In frame mode, once a complete, error free RTCM message has been received by the server application, a data frame containing the individual RTCM message and other additional data is created and transmitted. In the raw mode, the byte set that constitutes an RTCM message is transmitted by the server application (at the same rate it is generated by the DGPS base station) without any additional processing or verification. This latter mode was implemented in order to provide compatibility with other existing end-user Internet DGPS data clients. The DGPS Data Campus Server expects to receive DGPS data in frame mode.

\section{A.1.1 Frame Mode}

When in frame mode, the server application creates a data frame for each individual RTCM message received. In order to obtain a RTCM message from the information generated by a DGPS base station, the server application has to process the received data. The DGPS base station outputs a continuous byte stream via the serial interface that must be collected, decoded and assembled into valid RCTM messages.

The byte stream received through the serial interface needs appropriate processing for several reasons: (i) each 8-bit byte only contains 6 RTCM bits; (ii) the 6 RTCM bits arrive in reverse order (least significant bit first); (iii) every 30 bits of RTCM data holds 6 parity bits that must be checked in order to validate and decode the RTCM data.

The RTCM messages are made of RTCM words. Each RTCM word is 30-bit long and contains 24 data bits and 6 parity bits. Since one 8-bit byte only contains 6 RTCM bits, each RTCM word occupies five 8-bit bytes. The two most significant bits of each 8 -bit byte (the 2 non-RTCM bits should be set to space and mark (01), respectively.

The RTCM messages include, not only, 6 parity bits in each data word but, also, the data in the following data word is encoded according to the last 2 parity bits of the previous data word. The algorithm used to compute/verify the parity and to decode/encode the data bits is the algorithm specified for the GPS signal messages (for details please consult [6]).

Each RTCM message is composed by a mandatory header ( 2 word long), followed by a body of data of variable word length. The header includes a fixed preamble (01100110) and several other fields: message type ( 6 bits), reference station ID (10 bits), parity ( 6 bits), modified $Z$-count (13 bits), sequence number ( 3 bits), length of frame ( 5 bits), station health ( 3 bits) and a last parity field ( 6 bits). Once a correct preamble has been received, the next step is to find out about the length and the modified Z-count time of the incoming RTCM message. As the RTCM words are collected, the parity is permanently being verified. When a whole RTCM message is received, the data frame containing the RTCM message is created and sent over the established data link to the client.

Each data frame contains a header with a start of frame (SOF) byte, four bytes containing the DGPS server IP address, eight bytes holding the server time (ServT) at the moment the frame is created, eight bytes with the RTCM message modified Z-count time (MZC), a frame check sequence (FCS) byte and, finally, the RTCM message (Fig. 4).

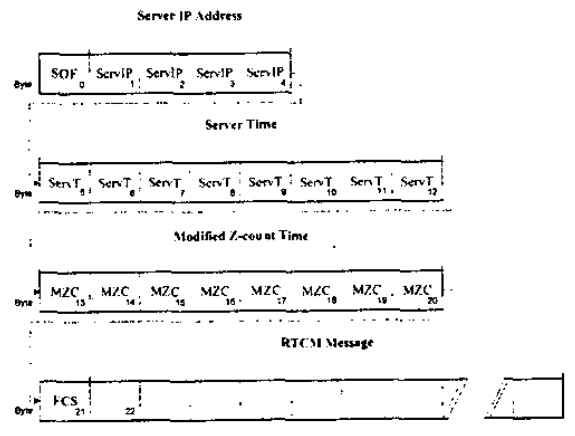

Fig. 4 - Data Frame.

In frame mode, the client-application receives the frames containing the RTCM-SC 104 messages and is immediately capable of verifying the quality of the data link transmission as well as the age of the messages being received. If the data frames were transmitted without errors and the messages they hold are still applicable, the data frames are forwarded to the DGPS data campus server application.

\section{B DGPS Data Campus Application}

The DGPS Data Campus application provides campus-wide DGPS data both via a radio beacon and via the campus Intranet (Fig. 5). The idea is to be able to use DGPS/GPS receivers with and without a DGPS data wireless interface [12]. The DGPS/GPS receivers without a DGPS data wireless interface have a RS232 DGPS input interface. In this latter case, a DGPS Data Campus Client 
can be installed, for example, in a laptop computer to receive the corrections data and to forward it via the RS232 interface to the DGPS/GPS receiver.

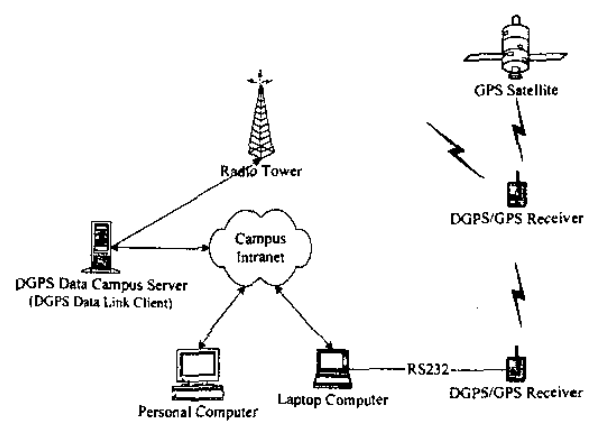

Fig. 5 - DGPS Data Campus Application.

The server data input module is the Internet Data Link Client. This input module is capable of establishing links with multiple remote DGPS servers using connection-oriented or the connectionless (message-oriented) links. Once a link is established, the client-side application receives the data either in frame or in raw transmission mode. When multiple data sources are used, additional processing is needed.

\section{B.1 DGPS Data Campus Server}

The DGPS Data Campus Server is composed of three functional units: the data input module (Internet Data Link client), the Intranet server and the wireless server (Fig. 6).

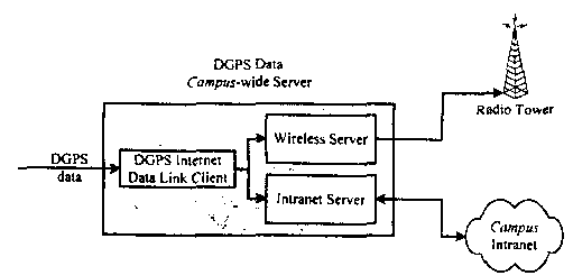

Fig. 6 - DGPS Data Campus Server.

The DGPS Data Campus Server receives the DGPS data directly from the DGPS Internet Data Link and forwards it immediately to both modules in order to minimize the transmission delay. The transmission delay is critical when using RTK corrections since the corrections update rate should not exceed the GPS update interval rate.

\section{B.1.1 Intranet Server Module}

The Intranet server module receives the data frames containing the RTCM messages, verifies the quality of the transmission and the age of the RTCM messages. If the data frames are error free and the messages are still applicable, the data frames are forwarded to all clients connected. The Intranet server multicasts DGPS data frames to all Intranet clients that join the specified multicast group at the application port created for this service. Currently, the datagrams time-to-live (TTL) parameter is set to one (just for the Electrical Engineering Department sub-network).

The implemented DGPS Intranet Server establishes simplex, point-to-multipoint, message-passing type of communication. The distributed application relies on the transport layer User Datagram Protocol (UDP) to provide a multicast service within the campus Intranet. Although UDP has no flow control mechanism, it is a message-oriented protocol, i.e., provides dynamic allocation of network bandwidth. In our case, since we need to establish simultaneously several connections within the local campus network, we adopted a multicast type of communication.

\section{B.1.2 Wireless Server Module}

In our case, the client application receives the DGPS corrections via the Internet link from the DGPS base stations and feeds them to the wireless server. The role of the wireless server is three fold: first, it formats and buffers the DGPS messages, then, it modulates the data onto the transmitter carrier and, finally, broadcasts the resulting data

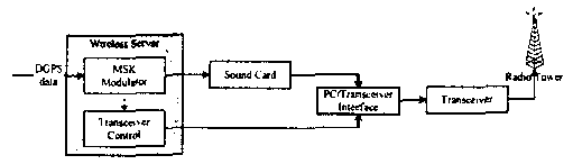

Fig. 7 - Wireless Server.

The data modulator receives the RTCM correction messages, encodes them as digital information using a Minimum Shift Keying (MSK) encoding algorithm, and forwards them to the computer sound card. Some special care must be taken in this processing since the RTCM protocol uses 30-bit length words and divides them into 6-bit length bytes that are sent to the modulator in standard IRA (ASCII) 8-bit bytes occupying the six least significant bits. The modulator strips the start, stop and the two most significant bits from this byte and only transmits the six RTCM SC-104 data bits over the air. At this point the data consists of just the RTCM SC-104 data protocol and the relationship between the start of a RTCM 30-bit word and an asynchronous IRA byte has been lost. Every time RTCM data is sent to the sound card, the transceiver control unit sends an automatic Push-To-Talk (PTT) command to the radio transceiver so that the RTCM data gets broadcasted.

\section{B.1.3 Wireless Data Link}

DGPS radio beacons modulate the carrier with the correction data and other information such as the health of the reference station and the identification of the transmitter. The modulation is Minimum Shift Keying (MSK), a special form of Frequency Shift Keying (FSK). In the case of pseudorange corrections, the modulation rate is usually $100 \mathrm{~b} / \mathrm{s}$ or $200 \mathrm{~b} / \mathrm{s}$. The radio regulations governing the use of this band are specified in the 
International Telecommunications Union (ITU) Recommendation M.823 [10], incorporate the RTCM $\mathrm{SC}-104$ protocol and allow the transmission of supplementary information (such as the DGPS signals).

The range of an individual transmitter is a function of power output, antenna height and efficiency, as well as the characteristics of the surrounding land. The broadcast data consists of a selected subset of the message types contained in the RTCM Special Committee No. 104, "Recommended Standards for Differential GNSS Service", Version 2.2, dated January 15, 1998 [11] and referred in this paper as "RTCM SC-104".

The corrections encoded as MSK and are then modulated onto the carrier of the radio beacon. MSK encoding results in approximately a $\pm 25 \mathrm{~Hz}$ shift in the carrier frequency of the radio beacon (at $100 \mathrm{~b} / \mathrm{s}$ ). During normal operation the minimum field strength of the DGPS broadcast signal will be $75 \mu \mathrm{V} / \mathrm{m}$ in the specified coverage area, at a transmission rate of $100 \mathrm{~b} / \mathrm{s}$.

\section{B.2 DGPS Data Campus Client}

The DGPS Data Campus Client is used to provide DGPS correction data to DGPS/GPS receivers without a wireless interface. In this case, the client-side application is used to establish the data link between the DGPS Data Campus Server and the serial interface of the receiver.

The client connects to DGPS Data Intranet Server by creating a multicast socket and by joining the service multicast session (multicast IP address of the host and the application port of the service). Once the link is established, the DGPS data frames are received. Whenever a data frame is error free and the RTCM message it holds is not too old, the message is immediately forwarded to the RS232 interface.

\section{STATUS AND RESULTS}

After some research, we found three DGPS base stations in the vicinity ( $10 \mathrm{~km}$ radius area) of our campus. At the time, none of the proprietary entities was broadcasting DGPS data in a format compliant with standard DGPS/GPS receivers.

One entity, herein called entity $A$, has a DGPS base station located at a distance of approximately $8 \mathrm{~km}$ to the northwest (NW) of our campus. The DGPS base station receiver has 12 single frequency (L1) tracking channels and generates RTCM V2.1 messages numbers 1 and 3 (pseudorange corrections). However, the radio broadcast frequency band (VHF) and the technology used are not compliant with standard GPS/DGPS receivers.

Entity $B$ runs a DGPS base station located at approximately $6 \mathrm{~km}$ to the south (S) of our campus and was collecting DGPS data only for post-processing (12 hours RINEX data files). Base station $B$ has 12 dual frequency ( $\mathrm{L} 1$ and $\mathrm{L} 2$ ) tracking channels and is capable of outputting RTCM V2.1 and V2.2 messages numbers 1, 2, $3,9,18,19,20,21$ and 22 (RTK and pseudorange corrections). Currently, as a result of this project, base station $B$ is already generating real time DGPS data (RTCM V2.1 and V2.2 messages numbers 1, 2, 3, 20, 21 and 22). Now, we are waiting for installation of the network cable between the base station and the nearest Internet node.

The third entity, the "Instituto para o Desenvolvimento Tecnológico" (IDT), is located at our campus and runs a DGPS base station equipped with a Trimble Pathfinder Pro XR GPS receiver with 12 single frequency (L1) tracking channels which can provide RTCM V2.1 messages numbers 1 and 3 (pseudorange corrections). When this project started, IDT provided only near real time data (hourly RINEX data files for post-processing). As a result of this project, the IDT base station is now a real time DGPS base station.

Currently, we are running a series of tests using the IDT base station as the sole data source. Since it only provides differential pseudorange corrections, we can only provide positioning results with this type of corrections. As soon as we have an Internet connection with entity B, we will be able to show results using RTK corrections.

The tests were carried out using two Garmin GPS/DGPS receivers: a Garmin 176 (G176) and a Garmin 35 (G35). Both receivers accept DGPS correction via the serial interface. To compare the accuracy obtained with and without the application of the pseudorange DGPS corrections, the receivers remained static through out the test and were positioned side by side.

While the G35 only received GPS data, the G176, which was connected via the serial interface to a DGPS Data Campus Client, received both GPS and DGPS data.

In the absence of differential corrections (Fig. 8), the latitude, longitude and elevation standard deviation values were $1.538 \mathrm{~m}, 1.335 \mathrm{~m}$ and $2.561 \mathrm{~m}$. With the application of differential corrections (Fig. 9) during the same period of time, these values dropped to $0.899 \mathrm{~m}, 1.023 \mathrm{~m}$ and $2.042 \mathrm{~m}$, respectively.

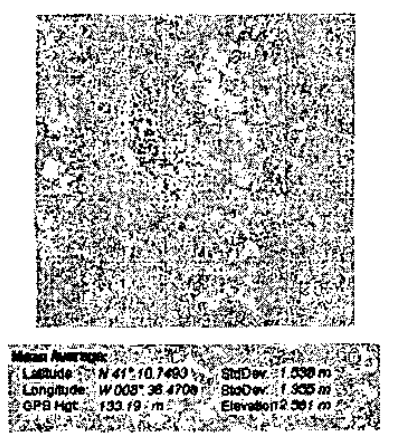

Fig. 8-G35 without differential corrections.

While in the case of the $\mathrm{G} 35$ receiver, the mean average positioning values were $41^{\circ} 10.7493 \mathrm{~N}, 8^{\circ} 36.4708 \mathrm{~W}$ and 
$133.19 \mathrm{~m}$ (elevation), in case of the G176, they were $41^{\circ} 10.7467 \mathrm{~N}, 8^{\circ} 36.4713 \mathrm{~W}$ and $132.60 \mathrm{~m}$ (elevation).

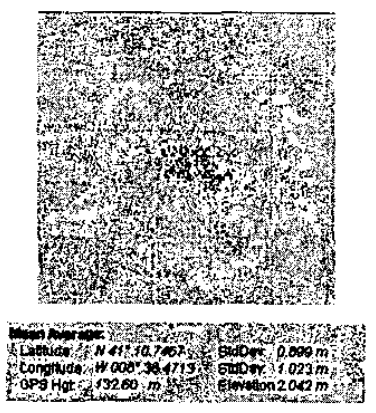

Fig. 9-G176 with differential corrections

The positioning results obtained are shown using VisualGPS, a copyright tool for displaying GPS positioning data from VGPS [16].

Additionally, we have also used existing on-line DGPS servers located in other parts of the globe. We were able to establish successful data links between our DGPS campus-server application and these remote data sources. Although the data received was not appropriate for our campus (the received corrections were for satellites out of sight), it was possible to verify the compatibility between systems.

We are running intensive tests, collecting system performance indicators and hope to be able to provide a detailed system evaluation in a near future. So far, we have been able to verify that the implemented functionalities are working and that the data collected supports this claim.

Meanwhile, the automatic control of the transceiver and the implementation of the PC/transceiver interface have been tested with success.

\section{RELATED WORK}

The idea of disseminating RTCM corrections over the Internet in real time for precise differential positioning and navigation purposes was also investigated by $\mathrm{W}$. Rupprecht [15]. In 1999, Rupprecht developed a DGPS data server called DGPSIP that disseminated DGPS data received through a radio interface at an average of $284 \mathrm{~b} / \mathrm{s}$. The radio was (and still is) normally tuned to POINT BLUNT, CA, Coast Guard transmitter: the server sends out a packet roughly every second with 35 bytes of data and 40 bytes of IP header. The DGPSIP is a multithreaded server, supports up to 64 concurrent connections and also transmits multicast UDP corrections (currently on 224.0.1.235 port 2101).

In 2002, the EUREF (European Reference Frame) which is a Sub-Commission of IAG's (International Association of Geodesy) Commission X on Global and Regional Geodetic Networks, decided to set up and maintain a differential GNSS infrastructure (DGNSS) on the Internet using stations of its European GPS/GLONASS
Permanent Network (EPN). The objective was to disseminate RTCM corrections over the Internet in real time for precise differential positioning and navigation purposes [5]. The acronym for these activities is EUREF-IP (IP for Internet Protocol). DGNSS trial servers currently provide RTCM corrections as generated in a number of European countries. The EUREF provides since 2002 free client software to access the appropriate data streams. This implementation transmits the DGPS corrections through the Internet using the standard RTCM SC-104 protocol over TCP.

More recently, several research teams have proposed and designed systems that use of GPRS as yet another support technology for the dissemination of DGPS data.

\section{CONCLUSION}

In order to have access, within our campus, to DGPS correction data, we developed a three-tier client/server distributed application. The first tier - the client tier represents the end client applications (wireless or Intranet clients located within the campus). The intermediate layer - the campus server - fetches and disseminates the DGPS data for the end client applications. The third layer - the servers of DGPS data - acts as the data provider layer to the intermediate layer.

The first and third layers are insulated. This approach prevents the potential congestion of the data source servers and allows the adoption of different transport protocols between the first and second layers and between the second and first layers.

As a result, for the campus data dissemination we use multicast and for the Internet data links we provide both connection-oriented (TCP) and message-oriented (multicast) types of communication.

Furthermore, the data can be sent in frame mode or in raw mode. Whereas the byte stream mode was kept for reasons of compatibility with existing DGPS client software applications (for example, the client software provide by [5] and [15]), the frame mode was designed to allow the simultaneous connection with multiple DGPS sources and to provide an almost instantaneous way of checking if the transmission was error free and of getting the age of the RTCM message.

The preliminary results we have gathered so far have shown that the service provided by the overall distributed system provides low-cost support for accurate outdoor campus navigation. We expect to further increase the positioning accuracy within our campus as soon as we have access to a differential RTK data source (entity B). Our current work is focused on testing and evaluating the system herein described.

\section{AKNOWLEDGEMENTS}

The authors wish to thank the following entities: 
- Administração dos Portos do Douro e Leixð̃es

- Instituto Geográfico Português

- Observatório Astronómico Prof. Manuel de Barros

- Instituto para o Desenvolvimento Tecnológico

- Instituto Superior de Engenharia do Porto.

\section{REFERENCES}

[1] Chuck Gilbert, Comparison of Commercial and Government RTCM Sources, Earth Observation Magazine, Volume 4, Number 3, http://www.eomonline.com/Common/Archives/M arch95/gilbert.htm, 1995.

[2] Chuck Gilbert, Differential Global Positioning System (DGPS) Broadcast Standard For Marine Navigation,

http://www.ccg-gcc.gc.ca/dgps/main_e.htm, 2002.

[3] DOD, NAVSTAR GPS User Equipment (Public Release Version), GPS Joint Program Office, Feb. 1991.

[4] Elliott D. Kaplan Editor, Understanding GPS. Principles and Applications, Artech House Publishers, 1996.

[5] EUREF, EUREF - Real Time, http://igs.ifag.de/euref realtime.htm, 2002.

[6] Global Positioning System, Standard Positioning Service Signal Specification, GPS, $2^{\text {nd }}$ Edition, 1995.

[7] Hofmann-Wellenhof, B., Lichtenegger, H., and Collins, J., GPS: Theory and Practice, Springer-Verlag Wien, 1994.

[8] Leick, Alfred, GPS Satellite Surveying, John Wiley \& Sons, Inc., 1995.

[9] Logsdon, Tom, Understanding The Navstar, 2nd Edit. Van Nostrand Reinhold, 1995.

[10] Recommendation ITU-R M.823-2, Technical Characteristics of Differential Transmissions for Global Navigation Satellite Systems from Maritime Radio Beacons in the Frequency Band of 283.5-315 k Hz in Region 1 and $285-325 \mathrm{kHz}$ in regions 2 and 3, 1997.

[11] RTCM Recommended Standards for Differential GNSS (Global Navigation Satellite Systems) Service, Special Committee N.104, Radio Technical Commission for Maritime Services, 1998.

[12] Soares, M. G., Malheiro, B., Restivo, F. J., Implementation of a Campus-Wide DGPS Data Server, Actas da $5^{a}$ Conferência sobre Redes de Computadores - CRC'2002, Faro, Portugal, Setembro, 2002.

[13] Soares, M. G., Restivo, F. J., Navegação em Tempo Real de Alta Precisão com Sistemas de Satélites, $1^{\circ}$ Fórum Tecnológico do IPP, Abril 1997.
[14] Soares, M. G., Restivo, F. J., Precisão em Navegação Contínua Terrestre e Maritima cm DGPS e GPSI, $3^{\circ}$ Encontro Nacional do Colégio de Engenharia Electrotécnico, Exponor-Matosinhos, Junho 1997.

[15] W. Rupprecht, DGPS corrections over the Internet, http:/www.wsrcc.com/wolfgang/gps/dgps-ip.htm 1, 2000.

[16] VisualGPS, Version 3.33, VGPS, http://www.visualgps.net/VisualGPS, 2002. 